\title{
Antibody gene transfer for HIV immunoprophylaxis
}

\author{
Alejandro B Balazs \& Anthony P West Jr \\ Antibody gene transfer, which involves the delivery of genes that encode potent, broadly neutralizing antibodies \\ to human immunodeficiency virus (HIV), is a promising new strategy for preventing HIV infection. A satellite \\ symposium at the AIDS Vaccine 2012 conference brought together many of the groups working in this field.
}

Da espite nearly 30 years of intense study, efforts to develop a safe and effective vaccine against human immunodeficiency virus (HIV) by conventional means have either failed or provided only modest, short-lived protection. Although the promising results of the trial of the HIV vaccine RV144 in Thailand continue to guide efforts to improve the efficacy and duration of a vaccine that harnesses the natural immune system, it remains uncertain when or if such efforts will succeed.

Studies describing the discovery of potent, broadly neutralizing antibodies that target HIV from chronically infected patients have demonstrated the potential for the humoral response to produce protective antibodies during the course of natural infection ${ }^{1,2}$. However, it is unclear whether immunogens can be designed that will elicit these rare antibodies efficiently.

The transfer of genes encoding antibodies ('antibody gene transfer') is a novel protective strategy that bypasses the natural immune response, which has been the central focus of previous attempts to develop a vaccine against HIV, by directing the production of antibodies from nonhematopoietic tissues, such as muscle (Fig. 1). Because this approach skips many of the steps in the usual path of vaccine development, it has been described as a 'leapfrog' strategy. Advances in the use of gene transfer for the correction of genetic deficiencies $^{3,4}$, particularly the successful expression

Alejandro B. Balazs and Anthony P. West Jr. are in the Division of Biology, California Institute of Technology, Pasadena, California, USA.

e-mail: apwest@caltech.edu of factor IX in a small group of patients with hemophilia $\mathrm{B}$, have bolstered the intriguing possibility of using adeno-associated virus (AAV) vectors as a vehicle for antibody gene delivery in humans. Two studies have demonstrated the feasibility of this approach against both simian immunodeficiency virus (SIV) in macaques ${ }^{5}$ and HIV in humanized mice ${ }^{6}$.

The Foundation for Vaccine Research organized a special satellite symposium at the AIDS Vaccine 2012 conference in September in Boston to discuss the latest developments in this promising area of translational research.

\section{AAV-mediated delivery of antibodies}

To provide a framework for the session, Phil Johnson (Children's Hospital of Philadelphia) delivered a comprehensive introduction to the biology and history of AAV vectors. A member of the parvoviridae family, AAV is a ubiquitous commensal virus in humans that has never been associated with any disease. It consists of a protein capsid shell that surrounds a single strand of genomic DNA that contains just two viral genes (which encode proteins that control viral replication and form the capsid), flanked on either side by inverted-terminal repeat (ITR) sequences. The ITR sequences form unique hairpin structures that, in conjunction with the two proteins, mediate both DNA replication and packaging during production of the virus. The natural propagation of AAV is entirely dependent on coinfection with adenovirus to deliver the necessary helper factors in trans. For the production of AAV vectors for immunoprophylaxis, a transgene encoding the desired antibody is inserted between two ITR sequences and can be packaged into viral particles by cotransfection with plasmid expressing the desired genes encoding replication and capsid proteins, as well as a separate plasmid to provide adenoviral helper functions. The serotype of the AAV vector produced is the result of the capsid-encoding gene, which affects both the cellular tropism of the vector and biological properties such as intracellular trafficking. AAV serotypes 1 and 8 have both been found to efficiently infect muscle tissues to form episomal head-to-tail concatemers of their genome in the nucleus, which results in abundant gene expression of very long duration, in animal models and have demonstrated excellent safety and tolerability in human trials. Presenting followup data from his 2009 study of protection of macaques given AAV serotype 1 to deliver genes encoding anti-SIV immunoadhesins (chimeric, antibody-based molecules that combine the functional domain of a binding protein with immunoglobulin crystallizable fragment $(\mathrm{Fc})$ domains $)^{5}$, Johnson showed that monkeys expressing the immunoadhesin 4L6 have had sustained circulating concentrations of approximately $20 \mu \mathrm{g} / \mathrm{ml}$ of $4 \mathrm{~L} 6$ for the past 5 years with no adverse health effects after the four intramuscular injections given at the initiation of the study. These results are consistent with previous AAV studies of macaques that have demonstrated stable erythropoietin expression for over 6 years ${ }^{7}$ and raise the hope that similar longevity of immunoprophylaxis might be achieved in human patients.

Animal models of immunoprophylaxis After the first descriptions of the broadly neutralizing antibodies $2 \mathrm{~F} 5,4 \mathrm{E} 10, \mathrm{~b} 12$ and 
2G12, many passive-transfer studies were undertaken to determine the efficacy of these antibodies in preventing infection with SIV-HIV-1 hybrid virus (SHIV) in macaques. Since that time, newer neutralizing antibodies have been described with considerably higher potency in vitro, which has raised the possibility that lower in vivo concentrations might be sufficient to provide protection. Dennis Burton (The Scripps Research Institute) confirmed that possibility during the session by presenting early results of experiments in which PGT121 demonstrated remarkable protection in macaques. Monkeys given a dose of PGT121 of $5 \mathrm{mg}$ per $\mathrm{kg}$ body weight $(5 \mathrm{mg} / \mathrm{kg}), 1 \mathrm{mg} / \mathrm{kg}$ or $0.2 \mathrm{mg} / \mathrm{kg}$ had approximately $100 \mu \mathrm{g} / \mathrm{ml}$, $15 \mu \mathrm{g} / \mathrm{ml}$ or $2 \mu \mathrm{g} / \mathrm{ml}$ of the antibody, respectively, in the circulation 1 day after administration and just before intravaginal challenge with 300 half-maximal tissue culture infectious doses of the SHIV strain $162_{\mathrm{P} 3}$. At the vaginal surface, PGT121 was detected at a concentration of $0.9 \mu \mathrm{g} / \mathrm{ml}$ or $0.2 \mu \mathrm{g} / \mathrm{ml}$, respectively, in monkeys that received a dose of PGT121 of $5 \mathrm{mg} / \mathrm{kg}$ or $1 \mathrm{mg} / \mathrm{kg}$, and it was undetectable in monkeys that received a dose of $0.2 \mathrm{mg} / \mathrm{kg}$. After challenge, all monkeys that received a dose of $5 \mathrm{mg} / \mathrm{kg}$ or $1 \mathrm{mg} / \mathrm{kg}$ remained uninfected, whereas three of five monkeys that received a dose of $0.2 \mathrm{mg} / \mathrm{kg}$ were protected from challenge, despite a lack of detectable antibody at the vaginal surface. These results represent an improvement over original studies of the antibody b12, in which a dose of $25 \mathrm{mg} / \mathrm{kg}$ protected eight of nine monkeys from a similar challenge ${ }^{8}$.

Human-to-human mucosal transmission of HIV requires that the virus mobilize across substantial host barriers, which results in the initiation of most infections by only one or a handful of viruses ${ }^{9,10}$. Substantial effort has been directed toward understanding the unique characteristics of such transmitted founder strains of HIV that have succeeded in this process ${ }^{11}$. Although enhanced resistance to neutralizing antibodies has not been observed for these strains ${ }^{10,11}$, it has remained unclear whether infection by such strains in vivo would show resistance to neutralization. In follow-up work to his earlier study demonstrating robust protection of humanized mice against the chemokine receptor CXCR4tropic NL4-3 strain of HIV by vectored immunoprophylaxis (VIP) ${ }^{6}$, David Baltimore (California Institute of Technology) presented results in which humanized mice given VIP to express antibody b12 or VRC01 were challenged with the REJO.c transmitted founder strain. In this experiment, substantial protection against infection with REJO.c

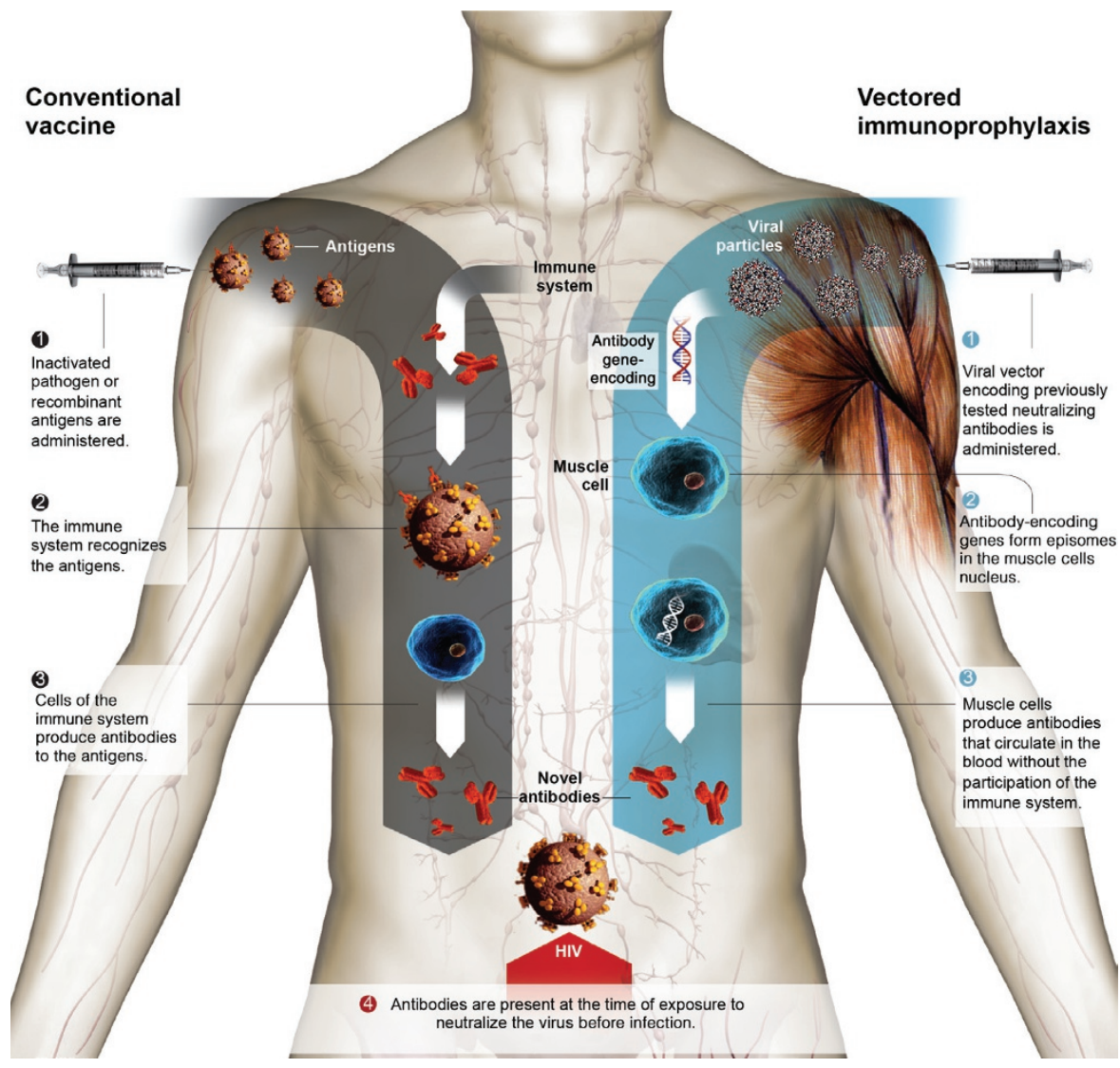

Figure 1 Comparison of prophylaxis approaches. Traditional vaccines work by engaging the adaptive immune system to produce a response that recognizes the administered antigen. VIP uses a viral vector such as AAV to deliver genes encoding a given antibody into muscle cells that then express the desired antibody and secrete it into the circulation without using the immune system. Adapted from an illustration prepared by La Vanguardia, Barcelona.

was noted in mice expressing VRC01 but not in those expressing b12, consistent with results obtained in vitro for these antibodystrain combinations. These results suggest that transmitted founder strains may not necessarily be more difficult to neutralize than nonfounder strains in vivo, which lends further support for the translation of the use of VIP to humans.

As most transmission of HIV occurs across mucosal surfaces, there have been considerable efforts to model this process in macaques and humanized BLT mice (bone marrow-liver-thymic chimeras) ${ }^{12,13}$. In addition, macaque studies have implemented repetitive challenge with a low dose of SIV to better simulate the low-probability transmission of HIV in humans ${ }^{14}$. During the session, Baltimore presented data obtained with a novel, modified humanized BLT mouse model that incorporates repetitive intravaginal challenge with a low dose of either the chemokine receptor CCR5-tropic laboratory strain JR-CSF or the REJO.c transmitted founder strain. With this model, he demonstrated that BLT mice given VIP to express either VRC01 or the more potent VRC01-like antibody VRC07G54W were highly resistant to intravaginal challenge with HIV. Challenge of mice expressing VRC01 or VRC07G54W resulted an undetectable viral load in five of eight or twelve of twelve mice, respectively, despite at least 15 exposures to virus. Interestingly, he observed limited depletion of $\mathrm{CD}^{+} \mathrm{T}$ cells in the peripheral blood but substantial depletion of $\mathrm{CD}^{-} \mathrm{T}$ cells in mucosal tissues of control mice expressing luciferase, analogous to observations noted for recently infected human patients ${ }^{15}$. Viral loads (assayed throughout the experiment by quantitative PCR) showed that control mice expressing luciferase were infected within six challenges with JR-CSF, whereas the two mice expressing VRC01 that became infected did so only at very late time points; this suggests that expression of VRC01 provides substantial protection from mucosal transmission. More strikingly, all of the mice expressing VRC07G54W were protected against as many as 20 consecutive weekly challenges with the REJO.c transmitted molecular founder strain and showed no signs of viral load by a com- 
mercial viral load assay with a sensitivity of 200 copies of viral RNA per ml.

\section{Challenges for prophylactic proteins}

Many studies have described the potential for proteins with non-natural antibody-based architectures to neutralize HIV. Among these architectures, immunoadhesins that consist of the fusion of a single-chain variable (Fv) fragment to an immunoglobulin G (IgG) crystallizable $(\mathrm{Fc})$ fragment domain have demonstrated potent activity in a macaque SIVchallenge model ${ }^{5}$. This architecture has the advantage of a single, compact coding region that enables delivery by self-complementary AAV vectors, which have limited carrying capacity ${ }^{5}$. However, studies have reported that the conversion of full-length antibodies into immunoadhesins can have a negative effect on neutralization potency ${ }^{16}$. Phil Johnson presented results comparing an immunoadhesin form of the antibody PG9 with the native IgG architecture in which he found that the native IgG architecture had a neutralization potency tenfold greater than that of the immunoadhesin. As a result of this, Johnson suggested that researchers might prefer natural antibody architectures for further clinical development.

The antibody PG9 recognizes a V2-V3 epitope on the HIV envelope trimer, and its activity is highly dependent on the presence of specific glycans ${ }^{17}$. The original description of this family of antibodies noted incomplete neutralization of certain viral strains regardless of antibody concentration ${ }^{1}$, which was subsequently ascribed, at least in part, to gly- can heterogeneity of the envelope spike protein. To determine the extent of this, Burton compared data from experiments testing a variety of antibodies to large virus panels. He concluded that the incomplete neutralization, observed in some cases, was most apparent for PG9-like and MPER antibodies but was relatively rare for many PGT antibodies that recognize glycans, as well as for antibodies to the CD4-binding site. Ultimately, the importance of viral glycan heterogeneity for antibody gene transfer is uncertain, but it probably should be factored into the choice of antibody combinations selected for prophylaxis.

\section{Improved antibodies}

Before the latest patient-derived broadly neutralizing antibodies reinvigorated the field, soluble bi- or tetravalent forms of CD4 were among the most potent protein-based anti-HIV reagents. Michael Farzan (Harvard University) presented studies on the fusion of such a reagent with a coreceptor mimetic peptide.

The HIV-1 coreceptors CCR5 and CXCR4 share amino-terminal domains that include sulfotyrosines, which bind to a conserved site on the HIV glycoprotein gp120. The heavychain complementarity-determining region 3 (CDR3) of several antibodies that target the coreceptor-binding site of gp120 also include sulfotyrosines, and sulfopeptides based on those CDR3 domains bind gp120 and neutralize HIV-1. A fusion of one such peptide with CD4-immunoglobulin, called 'eCD4-Ig', was at least as potent as the present genera- tion of antibodies to HIV and neutralized not only all neutralization-resistant (all tier 2 and tier 3) HIV-1 strains but also both HIV-2 and SIV isolates. Additionally, it substantially outperformed CD4-immunoglobulin and the antibody b12 in an assay of antibody-dependent cell-mediated cytotoxicity.

The dramatic breadth of the efficacy of eCD4-Ig presumably relates to its narrow targeting of HIV's conserved receptor- and coreceptor-binding sites, in contrast to antibodies, which generally rely on contacts with less-conserved regions of the HIV envelope protein. In the context of AAV-based gene delivery, eCD4-Ig is small enough to be delivered by self-complementary AAV vectors. Macaque studies are now being undertaken to determine the utility of eCD4-Ig in prophylactic and therapeutic contexts.

Pamela Bjorkman (California Institute of Technology) presented studies that have used structure-based design to improve the potency of antibodies to the CD4-binding site on gp120, as well as to overcome the common escape mutations the virus acquires to evade such antibodies. Antibody NIH45-46, a more potent variant of VRC01, contains an insertion that contacts the inner domain of gp120 and contributes to its enhanced activity relative to that of VRC01. Noticing that both NIH45-46 and VRC01 fail to fill a conserved hydrophobic pocket in gp120 that is filled by Phe 43 of CD4, the researchers designed a mutant of NIH45-46 with substitution of a tryptophan for the glycine at position 54, which they found to be about tenfold more potent than the parent antibody against a

\section{Box 1 An AIDS vaccine for wild chimpanzees?}

Beatrice Hahn (University of Pennsylvania) described the negative effect that the SIV strain SIVcpz has on wild chimpanzee populations. Although some SIV strains do not cause pathology in their natural hosts, Hahn presented evidence that chimpanzees infected with SIVcpz have a 10- to 16-fold higher risk of death and can develop pathology similar to that of end-stage AIDS. In wild chimpanzee populations already jeopardized by habitat loss and poaching, SIVcpz could pose a serious additional threat. Indeed, one chimpanzee community in Gombe National Park, Tanzania, where the prevalence of SIVcpz has consistently been over $40 \%$, has suffered a dramatic decrease in population size over the past four decades. Accompanying the loss of wild populations, such as those in Gombe, will be the loss of decades of behavioral and other research done at these unique sites.

HIV infection can be controlled by antiretroviral therapy; however, treating wild chimpanzees with daily medication is infeasible. Although there is no effective vaccine against SIV, AAV-mediated transfer of antibody-encoding genes offers a potential approach. This method might be practical for wild chimpanzees, because a single dose of AAV administered by dart could potentially be sufficient to induce long-lasting antibody expression. The success of VIP could be monitored noninvasively, with fecal screening for secreted antibodies and by testing for new infections with SIVcpz.

AAV-mediated gene delivery to control SIVcpz infection would require highly potent and broadly neutralizing anti-SIVcpz reagents. To identify such reagents, Hahn and colleagues used a panel of eleven SIVcpz infectious molecular clones, one SIVgor infectious molecular clone and three HIV-1 control infectious molecular clones to assess antibody neutralization. They screened nearly 50 monoclonal antibodies known to broadly and potently neutralize HIV-1. Generally, these antibodies had little neutralizing activity on the panel of SIVcpz infectious molecular clones. The only effective neutralizers were reagents containing domains 1 and 2 from CD4, the best of which included a fused sulfopeptide as described in the presentation by Michael Farzan. These reagents may be considered for an antibodymediated vaccine for wild chimpanzees that could protect certain endangered populations from simian AIDS, and the outcome of VIP in chimpanzees may also inform human studies. 
cross-clade panel of difficult-to-neutralize viruses $^{18}$.

Highly potent VRC01-like antibodies, which have been isolated from at least five patients, derive from the same germline gene $\left(\mathrm{V}_{\mathrm{H}} 1-2^{\star} 02\right)$. Bjorkman described key shared features of these antibodies, including a short CDR L3 loop and characteristic heavy-chain residues (Trp50, Asn58, Arg71 and Trp100B). These residues are critical for initial binding of the germline $\mathrm{V}_{\mathrm{H}} 1-2^{*} 02$-encoded antibody to its HIV-1 target and explain the restricted use of gene segments encoding the variable heavy-chain region $\left(\mathrm{V}_{\mathrm{H}}\right)$ during the development of this class of antibodies ${ }^{19}$. The lack of genes with these features in laboratory animals suggests that attempts to test vaccines designed to induce these antibodies may need to use mice with human antibody-encoding genes.

Viruses resistant to VRC01-like antibodies often have substitutions at sites contacted by the aforementioned characteristic residues. That observation was used for the design of antibody variants to neutralize such viruses. One such antibody, 45-46m2, neutralized $96 \%$ of HIV strains in a cross-clade panel and neutralized a set of viral isolates resistant to all other known broadly neutralizing antibodies $^{20}$. A second variant, 45-46m7, designed to thwart resistance to the engineered antibody NIH45-46 ${ }^{\mathrm{G} 54 \mathrm{~W}}$, restores the neutralization of consensus escape mutants and thus effectively targets a common route of viral escape from this class of antibodies. Bjorkman emphasized that finding a few well-chosen substitutions able to substantially improve natural broadly neutralizing antibodies demonstrates that such antibodies are not necessarily optimal as isolated, which raises the possibility of continued improvements by structure-based design methods. In addition, raising the frequency of viral isolates neutralized from $\sim 90 \%$ (VRC01 and related antibodies) to $96 \%$ suggests that achieving a breadth of nearly $100 \%$ may be possible.

\section{Antibodies as a treatment?}

Although the meeting dealt mainly with the potential for the delivery of antibodyencoding genes to prevent infection with HIV-1, Michel Nussenzweig (The Rockefeller University) presented studies exploring the possibility that antibodies might also be used to treat established infections ${ }^{21}$. Antiretroviral therapy has been a resounding success, but daily doses, side effects and resistance to antiretroviral drugs suggest that alternatives should be investigated.

Studies demonstrated that the first genera- tion of broadly neutralizing antibodies (b12, 2G12, 4E10 and 2F5) were of limited efficacy as therapeutics in humans or in humanized mice because escape variants emerged within a short period of time. To examine the potential of more-potent antibodies to function as therapeutic agents, the Nussenzweig laboratory has made use of humanized mice engrafted with hematopoietic stem cells and then infected with a CCR5-tropic HIV-1 strain of HIV 22 .

They treated groups of mice with either a single antibody (among five different broadly neutralizing antibodies) or combinations of three or five antibodies. They used the following antibodies that target several different epitopes: NIH45-46 G54W, PG16, PGT128, 10-1074 (a more potent variant of PGT121) and 3BC176 (which recognizes a conformational but as-yet-undefined epitope).

Nussenzweig reported that as in previous studies, for all of the antibodies tested, HIV-1 escaped rapidly from treatment with a single antibody. However, the viruses that escaped showed a limited number of genetic alterations encoding sites targeted directly by the antibodies. Moreover, they found that combinations of five broadly neutralizing antibodies effectively controlled HIV-1 infection and suppressed the viral load to below the limit of detection during the entire therapy period of up to 60 days. In contrast to the transient viral suppression observed after discontinuation of antiretroviral therapy ${ }^{23-25}$, the longer half-life of antibodies led to viremic control for an average of 60 days after cessation of therapy. Nussenzweig concluded that combinations of potent monoclonal antibodies can effectively control HIV-1 replication in humanized mice, and he suggested that such antibodies should be reexamined as a therapeutic modality in HIV-1-infected people.

\section{Prospects for human trials}

Given the encouraging results being reported by many groups for animal models of HIV transmission, there is considerable interest in accelerating the translation of such findings to both humans and animals through clinical trials (Box 1). However, this promising approach carries with it many unique challenges that must be addressed before proof of principle can be achieved in humans. Given the seemingly permanent nature of AAV transduction in animal models, there are heightened concerns about the choice of the antibody-encoding transgene used for immunoprophylaxis. Several groups have reported natural poly- or autoreactivity of some of the broadly neutralizing antibodies, and certain improvements in potency via structure-based design have resulted in enhanced autoreactivity. Although polyreactivity is not an uncommon feature of natural antibodies to $\mathrm{HIV}^{26}$, there are concerns about off-target toxicity and effect on antibody half-life. To address those concerns, evaluation of antibody proteins in humans by passive transfer to confirm safety and activity before delivery by viral vectors may be warranted. Despite such testing, the possibility of rare adverse events may spur the development of immunoprophylaxis vectors able to regulate or eliminate antibody expression in such cases. Ultimately, adverse events may be minimized as a result of improvements in antibody potency, which substantially diminish the serum concentrations needed to achieve sterilizing protection in animals. Potency improvements would have the added benefit of decreasing the potential costs of VIP by allowing the administration of lower vector doses to patients.

Two separate phase 1 trials now under development involve the use of AAV for the expression of antibody from muscle tissues. Phil Johnson, who is leading one of the trials, has completed discussions with the US Food and Drug Administration to conduct a trial of AAV serotype 1 expressing the antibody PG9 from muscle in high-risk, seronegative patients. Clinical-grade manufacture of this vector has been completed, and the trial is expected to commence in the near future. David Baltimore, in partnership with the Vaccine Research Center at the US National Institutes of Health, is planning a separate trial of AAV serotype 8 that expresses an antibody to the CD4-binding site from muscle in infected patients who are receiving treatment with antiretroviral drugs. During the session, he noted that efforts are now underway to identify the manufacturing capacity for vector production and to finalize the design of the trial.

Despite the challenges noted above, successful demonstration of gene transferbased immunoprophylaxis could alter the landscape of vaccine development and provide a new pathway for tackling challenging vaccine targets such as hepatitis $C$ virus, pandemic influenza virus and malaria. The ever-expanding universe of antibodies that target not only infectious diseases but also aberrant forms of endogenous proteins may lead to the development of entirely new prophylactic and therapeutic interventions that could have a substantial effect on protecting patients from disease.

\section{ACKNOWLEDGMENTS}

We thank session presenters for allowing descriptions of unpublished work, and P. Hale for discussions ; S.A. Plotkin and B.H. Hahn for organizing the scientific 
program; and the Foundation for Vaccine Research for sponsoring the symposium. The Foundation for Vaccine Research thanks the Wellcome Trust and International AIDS Vaccine Initiative for support.

\section{COMPETING FINANCIAL INTERESTS}

The authors declare no competing financial interests.

1. Walker, L.M. et al. Science 326, 285-289 (2009).

2. Wu, X. et al. Science 329, 856-861 (2010).

3. Nathwani, A.C. et al. N. Engl. J. Med. 365, 2357-2365 (2011).

4. Maguire, A.M. et al. Lancet 374, 1597-1605 (2009).

5. Johnson, P.R. et al. Nat. Med. 15, 901-906 (2009).
6. Balazs, A. B et al Nature 481, 81-84 (2011).

7. Rivera, V.M. et al. Blood 105, 1424-1430 (2005)

8. Hessell, A.J. et al. Nature 449, 101-104 (2007).

9. Salazar-Gonzalez, J.F. et al. J. Virol. 82, 3952-3970 (2008).

10. Keele, B.F. et al. Proc. Natl. Acad. Sci. USA 105, 7552-7557 (2008).

11. Wilen, C.B. et al. J. Virol. 85, 8514-8527 (2011).

12. Sun, Z. et al. J. Exp. Med. 204, 705-714 (2007).

13. Denton, P.W. et al. PLoS Med. 5, e16 (2008).

14. McDermott, A.B. et al. J. Virol. 78, 3140-3144 (2004).

15. Guadalupe, M. et al. J. Virol. 77, 11708-11717 (2003).

16. West, A.P., Galimidi, R.P., Gnanapragasam, P.N.P. \& Bjorkman, P.J. J. Virol. 86, 195-202 (2012).
17. Doores, K.J. \& Burton, D. R. J. Virol. 84, 10510-10521 (2010).

18. Diskin, R. et al. Science 334, 1289-1293 (2011)

19. West, A.P., Diskin, R., Nussenzweig, M.C. \& Bjorkman, P.J. Proc. Natl. Acad. Sci. USA 109, E2083-E2090 (2012).

20. Sather, D. N. et al. J. Virol. 86, 12676-12685 (2012).

21. Klein, F. et al. Nature advance online publication, doi:10.1038/nature11604 (24 October 2012).

22. Baenziger, S. et al. Proc. Natl. Acad. Sci. USA 103 15951-15956 (2006).

23. Choudhary, S.K. et al. J. Virol. 83, 8254-8258 (2009). 24. Nischang, M. et al. PLoS ONE 7, e38853 (2012)

25. Denton, P.W. et al. J. Virol. 86, 630-634 (2012).

26. Mouquet, H. et al. Nature 467, 591-595 (2010). 